Check for updates

Cite this: RSC Adv., 2017, 7, 19808

Received 26th February 2017

Accepted 28th March 2017

DOI: $10.1039 / \mathrm{c} 7 \mathrm{ra0} 2401 \mathrm{k}$

rsc.li/rsc-advances

\section{Enhanced fluorescence properties of terbium complex/poly-L-lactic acid superfine fibers sensitized by the LSPR effect of silver nanoparticles $\uparrow$}

\begin{abstract}
Shipeng Wen, (DD a Guiming Zhang, ${ }^{a}$ Rong Zhang, ${ }^{a}$ Liqun Zhang ${ }^{a}$ and Li Liu*ab
In this research, Ag-NPs/Tb-complex/PLLA composite fibers with diameters of around $250 \mathrm{~nm}$ were successfully obtained by the electrospinning and characterized by scanning electron microscope (SEM), transmission electron microscopy (TEM), X-ray photoelectron spectroscopy (XPS) and fluorescence spectra. The influence of localized surface plasmon resonance (LSPR) of silver nanoparticles (Ag-NPs) on the fluorescence of $\mathrm{Tb}(\mathrm{acac})_{3}$ phen/poly-L-lactic acid (Tb-complex, acac = acetylacetone; phen = phenanthroline, PLLA = poly-L-lactic acid) fibers was mainly investigated. It is found that Tb-complex dispersed in the fibers in the form of nanoclusters. The local field surrounding of $\mathrm{Tb}^{3+}$ ions was affected by the neighboring Ag-NPs. As a result of the LSPR effect of Ag-NPs, the fluorescence intensity, quantum efficiency of Tb-complex in the composite fibers with the Ag/Tb ratio of $4: 1$, were simultaneously improved compared with the composite fibers without Ag-NPs.
\end{abstract}

\section{Introduction}

Fluorescence nanofibers have attracted growing interest because of the potential applications in biological fields such as skeletal muscle tissue engineering, ${ }^{1}$ bio-imaging, ${ }^{2}$ drug carriers, ${ }^{3}$ and fluorescent probes. ${ }^{4}$ Traditional fabrication methods of fluorescence fibers mainly include solution spinning and melting spinning. Nevertheless, both of them can barely obtain the fibers with diameters below $500 \mathrm{~nm}$. In recent decades, electrospinning as a universal and effective method for fabricating uniform ultrafine fibers has exhibited many advantages, such as small diameters ranging from tens to hundreds of nanometers, ${ }^{5}$ high porosity, large specific surface area, ${ }^{6}$ large length to diameter ratios, and good uniformity. ${ }^{7}$ Also, electrospinning is a direct method of generating composite nanofibers containing luminescent agents with uniform dispersion. ${ }^{8}$ At present, many researchers have prepared fluorescence nanofibers via electrospinning. For instance, Zhang et al. ${ }^{9}$ prepared efficient luminescent $\mathrm{Eu}(\mathrm{TTA})_{3}$ phen/polystyrene (TTA $=2$-thenoyltrifluoroacetone, phen $=1,10$-phenanthroline) nanofibers with diameters ranging from 350 to $700 \mathrm{~nm}$ by electrospinning. Huang et al. ${ }^{\mathbf{1 0}}$ prepared $\mathrm{Eu}(\mathrm{DBM})_{3}$ phen/polystyrene $(\mathrm{DBM}=$ dibenzoylmethane) fluorescence composite fibers with

${ }^{a}$ Beijing Engineering Research Centre of Advanced Elastomers, Beijing University of Chemical Technology, Beijing 100029, China. E-mail: liul@mail.buct.edu.cn

${ }^{b}$ State Key Laboratory of Chemical Resource Engineering, Beijing University of Chemical Technology, Beijing 100029, China

$\dagger$ Electronic supplementary information (ESI) available. See DOI: $10.1039 / \mathrm{c} 7 \mathrm{ra} 02401 \mathrm{k}$ diameters of around $300 \mathrm{~nm}$ through electrospinning. The energy transfers from phen and DBM to $\mathrm{Eu}^{3+}$ ion were very efficient. The $\mathrm{Eu}^{3+}$ ions were located in a highly polarizable chemical environment, and this was beneficial to the fluorescence lifetime and internal quantum efficiency of the fibers. Gai et al. ${ }^{11}$ fabricated $\mathrm{Fe}_{3} \mathrm{O}_{4} / \mathrm{PVP} / / \mathrm{Tb}(\mathrm{BA})_{3}$ phen/poly(vinyl pyrrolidone) (BA = benzoic acid) magnetic-photoluminescence bifunctional nanofibers by employing a parallel axial electrospinning setup. The average diameter of the individual fiber was around $200 \mathrm{~nm}$. The nanofiber bundles exhibited strong green emissions under the excitation of $275 \mathrm{~nm}$.

It is known that the localized surface plasmon resonance (LSPR) effect is an optical phenomenon generated by collective oscillations of electron gas in metal nanostructures surrounded by a dielectric. ${ }^{12}$ As a typical material for plasmonic applications, silver displays sharp and intense LSPR bands. ${ }^{13}$ When the frequencies of incident light and the free electron collective oscillation are same, resonance can be produced. ${ }^{14}$ Adsorption of molecules on the metal surface lead to measurable spectral changes in both the wavelength and intensity of the LSPR. ${ }^{15,16}$

Surface plasmon resonance-enhanced fluorescence (SPREF) phenomenon of rare-earths/Ag hybrid can be generated when rare-earth particles are close to the Ag particles (Ag-NP) within a few nanometers. ${ }^{17-19}$ With the irradiation of visible light, the LSPR of Ag particles can be excited. The LSPR effect results in an enhanced electromagnetic radiation field and thus increases the emission intensity of rare-earth particles in the vicinity of the Ag particles. ${ }^{20,21}$ Previous research has confirmed that Ag-NP acts as an effective enhancement agent. ${ }^{22,23}$ Lin et al. ${ }^{24}$ prepared 
$\mathrm{Pb} / \mathrm{Cd}$-free oxyfluoride glasses, and then introduced molecularlike (ML) Ag clusters with 1-4 nm into the glasses. The results demonstrated that the energy transfer from ML-Ag clusters to $\mathrm{Tb}^{3+}$ and $\mathrm{Yb}^{3+}$ led to the enhancement of near infrared (NIR) emission intensity of $\mathrm{Yb}^{3+}$ ions. Hayakawa et al. ${ }^{25}$ prepared $\mathrm{SiO}_{2}$ glass containing $\mathrm{Ag}^{+}$and $\mathrm{Eu}^{3+}$ ions by annealing the glass in a reducing atmosphere. The results showed that an induced surface plasma oscillation by incidence and an interaction between $\mathrm{Eu}^{3+}$ and $\mathrm{Ag}$ particles occurs simultaneously. The fluorescence of $\mathrm{Eu}^{3+}$ was enhanced in the presence of silver particles with the size of $4.3 \mathrm{~nm}$. Zhang $e t$ al. ${ }^{26}$ prepared an Ag co-doped $\mathrm{SiO}_{2} \quad\left(\mathrm{SiO}_{2}: \mathrm{Tb}^{3+}-\mathrm{Ag}\right)$ nanostructured luminescent composite using a modified Stöber method. The results showed that Ag nanoparticles greatly enhanced the light absorption at $226 \mathrm{~nm}$ and the light emission at $543 \mathrm{~nm}$ of the composite, and the fluorescence lifetime reduced with the increase of $\mathrm{Ag}$ concentration in $\mathrm{SiO}_{2}: \mathrm{Tb}^{3+}-\mathrm{Ag}$ composite.

In this research, the main objective is to prepare superfine fibers with strong fluorescence properties in the presence of $\mathrm{Ag}$ nanoparticles. Hence, terbium complex $\mathrm{Tb}(\mathrm{acac})_{3}$ phen ( $\mathrm{Tb}$ complex) emitting a strong green light, and Ag-NPs were introduced into poly-L-lactic acid (PLLA) matrix. Finally, the PLLA superfine fibers containing Ag-NPs and Tb-complex were prepared by electrospinning. The diameter of the composite fibers was around $250 \mathrm{~nm}$. Tb-complex had a fine dispersion in the PLLA fibers. The fluorescence intensity of fibers at $539 \mathrm{~nm}$ was greatly enhanced by Ag-NPs when the mole ratio of $\mathrm{Ag}$ and Tb-complex was $4: 1$. The composite fibers displayed higher quantum efficiency and longer fluorescence lifetime than the composite fibers without Ag-NPs.

\section{Experimental}

\section{Materials}

Terbium oxide $\left(\mathrm{Tb}_{4} \mathrm{O}_{7}\right.$, purity $\left.99.9 \%\right)$ was purchased from Grirem Advanced Materials Co., Ltd (Beijing, China). Silver nanoparticles (Ag-NPs) and polyvinylpyrrolidone (PVP, $M_{\mathrm{w}}=$ 1300 000) were purchased from Sigma-Aldrich Trading Co., Ltd (Shanghai, China). Poly-L-lactic acid (PLLA, $M_{\mathrm{w}}=70000$ ) was obtained from Guanghua Weiye industrial Co., Ltd (Shenzhen, China). Acetylacetone (acac), phenanthroline (phen), dichloromethane (DCM), $N, N$-dimethyl formamide (DMF), nitric acid $\left(\mathrm{HNO}_{3}\right)$, and anhydrous ethanol $\left(\mathrm{C}_{2} \mathrm{H}_{5} \mathrm{OH}\right)$ are all commercial reagents.

\section{Synthesis of $\mathbf{T b}(\mathbf{a c a c})_{3}$ phen (Tb-complex)}

$\mathrm{Tb}(\mathrm{NO})_{3}$ was obtained through the reaction of $\mathrm{Tb}_{4} \mathrm{O}_{7}$ and $\mathrm{HNO}_{3}$. $0.1 \mathrm{~mol}$ of $\mathrm{Tb}(\mathrm{NO})_{3}$ was dissolved in $\mathrm{C}_{2} \mathrm{H}_{5} \mathrm{OH}$. The $\mathrm{Tb}(\mathrm{NO})_{3}$ solution was magnetically stirred until the $\mathrm{Tb}(\mathrm{NO})_{3}$ was dissolved completely. Then, $0.3 \mathrm{~mol}$ of acac was first dissolved in $\mathrm{C}_{2} \mathrm{H}_{5} \mathrm{OH}$ and this mixture was then added into the $\mathrm{Tb}(\mathrm{NO})_{3}$ solution. After 1 hour, $0.1 \mathrm{~mol}$ of phen was dissolved in $\mathrm{C}_{2} \mathrm{H}_{5} \mathrm{OH}$. The phen solution was then added to the $\mathrm{Tb}(\mathrm{NO})_{3}$ solution. 3 hours later, white precipitates gradually appeared. Finally, the precipitate was dried at $60{ }^{\circ} \mathrm{C}$. The production efficiency was $53 \%$. The IR spectra of acac, phen and Tb-complex are shown in ESI (Fig. S1†). The characteristic peaks of $\mathrm{C}=\mathrm{O}$ and $\mathrm{C}-\mathrm{N}$ shift to the new position after the reaction, indicating that $\mathrm{Tb}^{3+}$ ions have coordination reactions with the $\mathrm{C}=\mathrm{O}$ of acac and two nitrogen atoms of phen.

\section{Preparation of Ag-NPs/Tb-complex/PLLA fibers}

PLLA was dissolved in DCM, and the resulting solution was magnetically stirred for 12 hours to form solution (I). Tbcomplex was dissolved in DMF, and the resulting solution was magnetically stirred for 15 minutes to form solution (II). A certain amount of Ag-NPs and PVP were added to DMF to form solution (III) that was treated under ultrasonic waves for 1 hour. Finally, the solutions of (I), (II) and (III) were mixed together and magnetically stirred for 1 hour. In the mixture solution, the PLLA content was $8 \mathrm{wt} \%$ and the Tb-complex content was 5 wt $\%$. The mixture solution was then transferred into an injection syringe and ready to be electrospun into fibers. The parameters of the electrospinning process are listed as follows: the feeding rate was $1.0 \mathrm{ml} \mathrm{h}^{-1}$; the distance from needle to collector was $15 \mathrm{~cm}$; the voltage was $15 \mathrm{kV}$; the speed of collecting drum was $300-400 \mathrm{rpm}$. The electrospinning was carried out for 2.5 hours in order to achieve a fiber membrane. Finally, a series of fibers with Ag-NPs/Tb-complex mole ratios of $0,1,2$, $3,4,5,8$ and 10 , were prepared separately.

\section{Characterizations}

The morphology of fibers was observed by a scanning electron microscope (SEM, S-4800); prior to observation, gold processing for the samples was received and the voltage was set at $1 \mathrm{kV}$. A transmission electron microscope (TEM, Tecnai G2 F30 STWIN) and an energy dispersive X-ray spectroscope (EDS) were used to observe dispersion of $\mathrm{Tb}$-complex and $\mathrm{Ag}$ particles in the fibers. An X-ray photoelectron spectrometer (XPS, Escalab 250) was used to detect the contents of $\mathrm{Ag}$ and $\mathrm{Tb}$ in different areas of the fibers. Excitation and emission spectra of the fibers were obtained from a fluorescence spectrophotometer (F-4500, Hitachi). The scanning speed, sweep voltage and slit width were set at $1200 \mathrm{~nm} \mathrm{m^{-1 }}, 400 \mathrm{~V}$ and $2.5 \mathrm{~nm}$, respectively. Fluorescence quantum efficiency was obtained from a fluorescence spectrophotometer (F-4700, Hitachi). An integrating sphere method was used to gain the latter's absolute rate. Wavelengths of excitation and emission of Tb-complex and fibers were set at $294 \mathrm{~nm}$ and $539 \mathrm{~nm}$, respectively. Neutral $\mathrm{Al}_{2} \mathrm{O}_{3}$ powders and empty fiber were used as the reference samples for the Tb-complex and fibers, respectively. Fluorescence lifetime was obtained by use of a steady-state and timeresolved fluorescence spectrometer (FLS920, Edinburgh Instruments). The excitation wavelength was set at $294 \mathrm{~nm}$.

\section{Results and discussion}

Original morphologies of Tb-complex particles are shown in Fig. 1A. From the inset, it can be seen that Tb-complex particles have a sheet shape. The size of most Tb-complex particles is in the range of $4-6 \mu \mathrm{m}$. Fig. 1B shows that Ag-NPs have a spherical shape with diameters of around $30 \mathrm{~nm}$, and are easily 

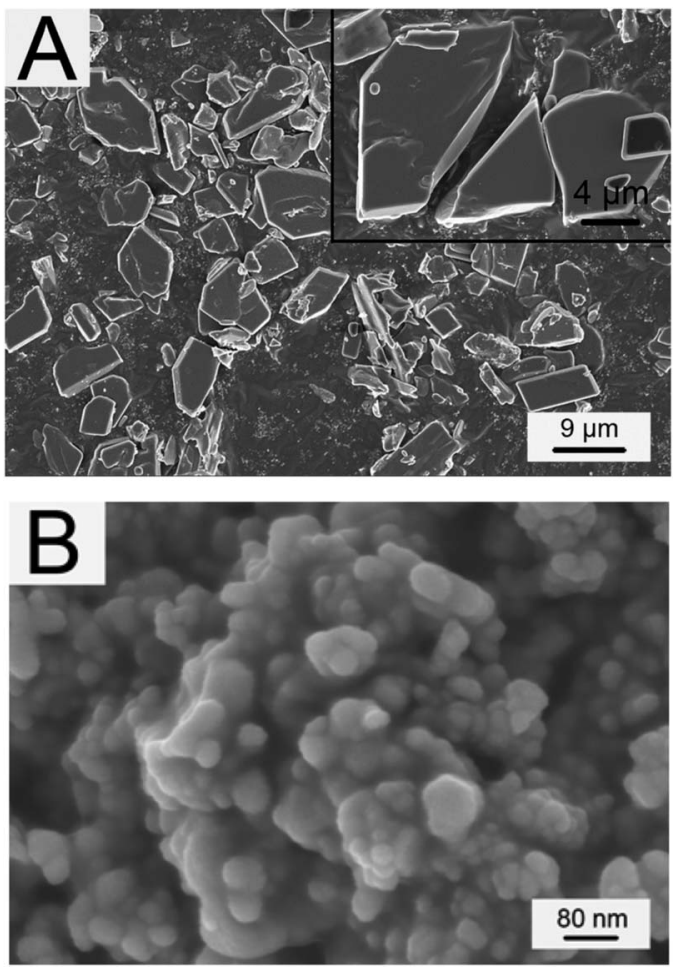

Fig. 1 SEM images of (A) Tb-complex particles and (B) Ag-NPs.

aggregated into a particle of larger dimension (Fig. 1B). Hence, PVP as dispersion agent was added into the $\mathrm{Ag}$ electrospun solution before electrospinning to ensure Ag-NPs were well dispersed in the solution.

Fig. 2 shows the morphologies of Tb-complex/PLLA and AgNPs/Tb-complex/PLLA fibers. Tb-complex/PLLA fibers have the diameters of about $400 \mathrm{~nm}$ and smooth surfaces (Fig. 2A). After Ag-NPs were added into the Tb-complex/PLLA fibers, the diameter of the fibers further decreased to about $250 \mathrm{~nm}$ (Fig. 2B). The reason for this decrease in fiber diameter is attributed to the higher charge density on the surface of ejected jet after the addition of Ag-NPs. The increased charge density further results in high elongation forces imposed to the jet under the electrical field. It is known that the overall tension in the electrospun fibers depends on the self-repulsion of the excess charges on the jet. Therefore, the increased charge density of fibers after the addition of Ag-NPs leads to thinner diameter of the fibers. ${ }^{27-29}$

TEM and EDS were further employed to observe the dispersion of Ag-NPs and Tb-complex particles in the fibers. Fig. 3A shows a TEM image of Tb-complex/PLLA fibers. Only some very tiny black dots of Tb-complex particles with the size under $5 \mathrm{~nm}$ can be observed in the interior of the fiber. EDS was used to detect $\mathrm{Tb}$ contents in different areas. The results show that the contents of $\mathrm{Tb}$ in area 1 and 2 are 4.23 and $4.76 \mathrm{wt} \%$, respectively. Compared with the large sheet shape of original $\mathrm{Tb}$ complex particles illustrated in Fig. 1A, the decrease in the size of Tb-complex particles in the fibers can be attributed to particular features of electrospinning process. Before the electrospinning process, Tb-complex was first dissolved in the

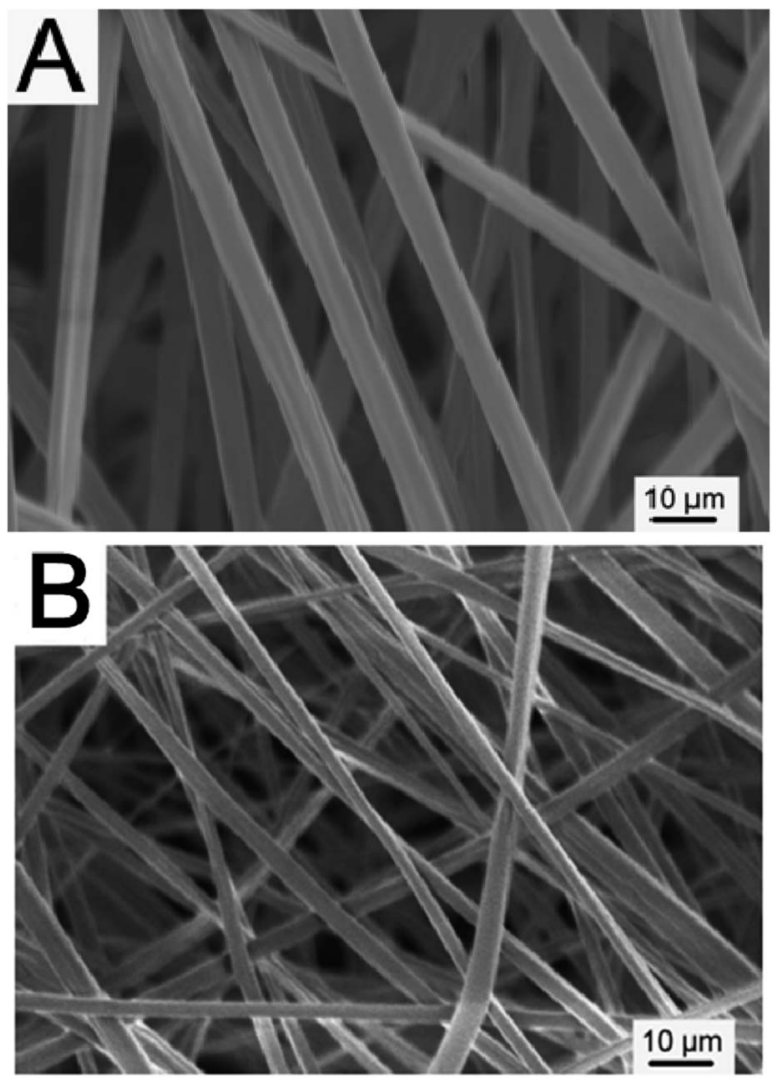

Fig. 2 SEM images of (A) Tb-complex/PLLA fibers, (B) Ag-NPs/Tbcomplex/PLLA fibers ( $\mathrm{Ag}: \mathrm{Tb}=4: 1$ ).

electrospun solutions and existed in the form of single molecule. During the electrospinning process, solvents in the electrospun solution evaporated quickly and the Tb-complex molecules did not have enough time to aggregate into big particles. As a result, most Tb-complex molecules may gather into some nanoclusters in the fibers. Fig. 3B shows the dispersion of Ag-NPs and Tb-complex in the fibers. Ag-NPs of different diameters are incorporated in the fibers such as in the range of $50-80 \mathrm{~nm}$ instead of original $30 \mathrm{~nm}$. That is because Ag-NPs are incompatible with PVP molecules, resulting in a certain degree of phase separation between them. Although ultrasonic treatment of Ag solution was employed before electrospinning, AgNPs still tended to form aggregates in the composite fibers. EDS detection was further used in a rich Ag-NP area and a blank area. The results show that the contents of $\mathrm{Tb}^{3+}$ ions in area 3 and 4 are 3.02 and $2.58 \mathrm{wt} \%$, respectively. This indicates that the addition of Ag-NPs do not affect the dispersion of $\mathrm{Tb}$ complex in the fibers.

XPS was used to investigate the influence of Ag-NPs on the local field surrounding of $\mathrm{Tb}^{3+}$ ions in the fibers. The element scans of XPS show that the contents of $\mathrm{Tb}^{3+}$ ions in the PLLA and Ag-NPs/Tb-complex/PLLA (Ag/Tb $=4: 1$ ) fibers are 3.20 and $3.32 \mathrm{wt} \%$, respectively. These results are consistent with those from TEM-EDS tests. In the XPS spectra (Fig. 4.) for Tb-complex/ PLLA fibers, both peaks appear at 1245.9 and $1225.1 \mathrm{eV}$ attributed to $3 \mathrm{~d}_{5 / 2}$ of $\mathrm{Tb}^{3+}$ ions. However, the two peaks in the XPS spectra for Ag-NPs/Tb-complex/PLLA fibers shift to a lower 

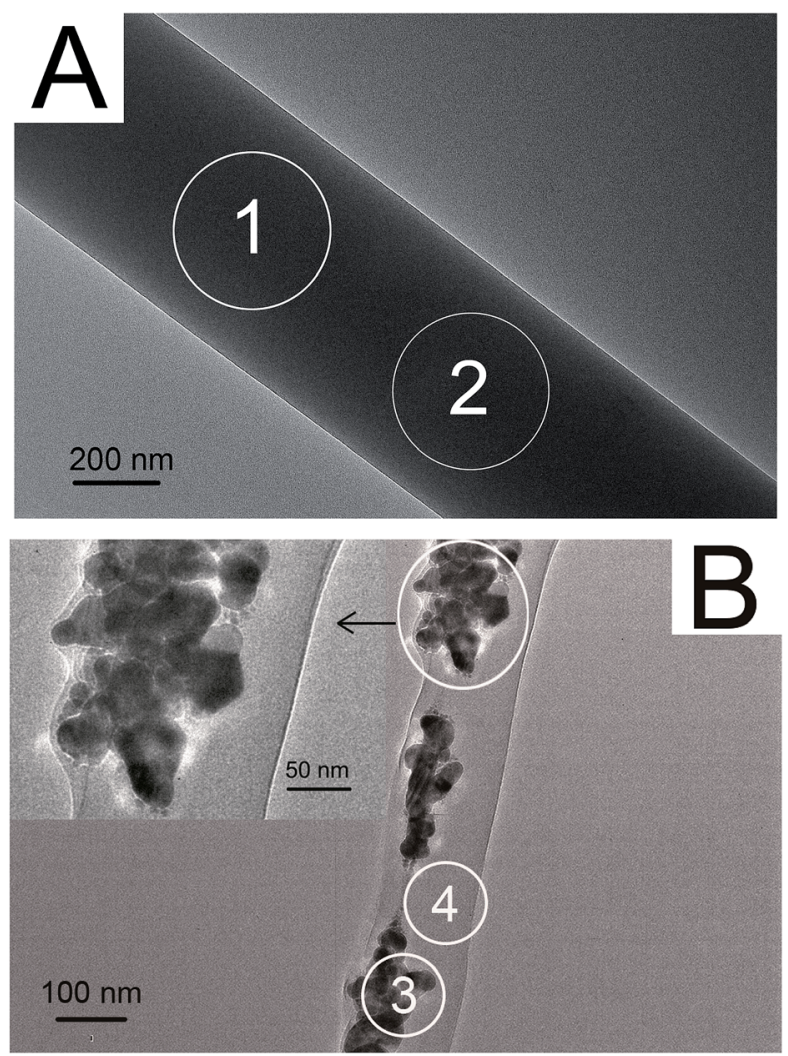

Fig. 3 TEM images of (A) Tb-complex/PLLA fibers containing $5 \mathrm{wt} \%$ $\mathrm{Tb}$-complex and (B) Ag-NPs/Tb-complex/PLLA fibers (Ag: Tb = $4: 1$ ).

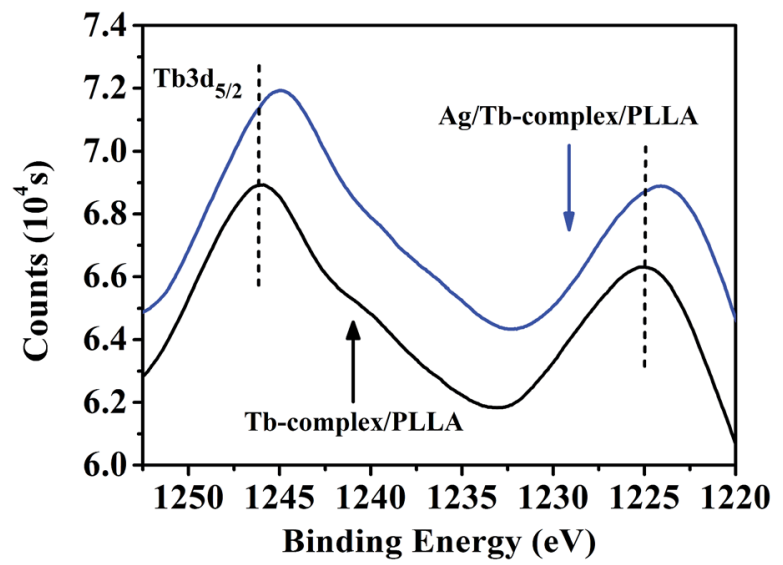

Fig. 4 Binding energy of $\mathrm{Tb}^{3+}$ ions in the Tb-complex/PLLA and Ag/ Tb-complex/PLLA fibers.

binding energy. This change indicates that Ag-NPs have changed the local field surrounding of $\mathrm{Tb}^{3+}$ ions in the fibers.

Fig. 5 shows the excitation and emission spectra of $\mathrm{Tb}$ complex. The excitation spectrum (Fig. 5A) exhibits a platform, revealing that the Tb-complex can be excited by a widerange light ranging from $325-360 \mathrm{~nm}$. The broad platform is mainly attributed to the wide absorption of organic ligands acac and phen. In the emission spectrum (Fig. 5B), there are four characteristic peaks corresponding to the ${ }^{5} \mathrm{D}_{4}{ }^{-}{ }^{7} \mathrm{~F}_{6}(490 \mathrm{~nm})$,
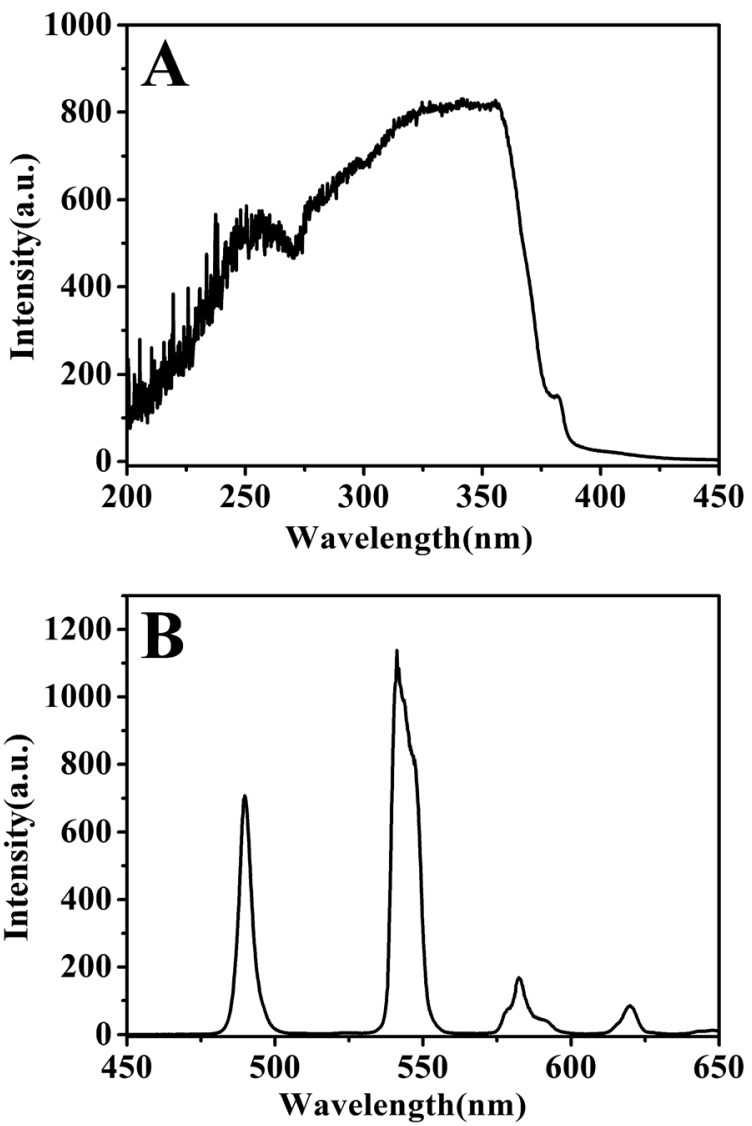

Fig. 5 (A) Excitation $\left(\lambda_{\mathrm{em}}=541 \mathrm{~nm}\right)$ and $(\mathrm{B})$ emission spectra $\left(\lambda_{\mathrm{ex}}=351\right.$ $\mathrm{nm}$ ) of Tb-complex.

${ }^{5} \mathrm{D}_{4}{ }^{-} \mathrm{F}_{5}(541 \mathrm{~nm}),{ }^{5} \mathrm{D}_{4}{ }^{-}{ }^{7} \mathrm{~F}_{4}(582 \mathrm{~nm}),{ }^{5} \mathrm{D}_{4}{ }^{-}{ }^{7} \mathrm{~F}_{3}(620 \mathrm{~nm}) \cdot{ }^{30}$ Among these peaks, the peak attributed to maximum emission intensity appears at $541 \mathrm{~nm}$. Good fluorescence performance is attributed to the high efficient energy transfer from the ligands acac and phen to $\mathrm{Tb}^{3+}$ ions.

Fig. 6 shows the excitation and emission spectra of Ag-NPs/ Tb-complex/PLLA fibers. It is noted that the broad peaks of Tb-complex in Fig. 5A have blue-shifted, and have been split into two peaks in composite fibers (Fig. 6A). This phenomenon indicates the local field surrounding around $\mathrm{Tb}^{3+}$ ions has changed in the fibers compared with that in Tb-complex powders. In the emission spectra (Fig. 6B), all samples show the same fluorescence peaks with Tb-complex illustrated in Fig. 5B. The strongest peak still appears around $541 \mathrm{~nm}$. The fluorescence intensity of fibers is much lower than that of $\mathrm{Tb}$ complex powders. The main reasons for the decreased fluorescence intensity are the low concentration of $\mathrm{Tb}^{3+}$ in the fibers and the poor absorption and transfer efficiency of the PLLA matrix. It should be noted that the fluorescence intensity of AgNPs/Tb-complex/PLLA fibers is stronger than that of Tbcomplex/PLLA fibers. As the content of Ag-NPs in the fibers increases (Fig. 6C), the fluorescence intensity of the composite fibers also increases and reaches the highest value when the mole ratio of $\mathrm{Ag}$ and $\mathrm{Tb}$ is $4: 1$. After reaching peak intensity, the fluorescence intensity begins to decrease. This result 

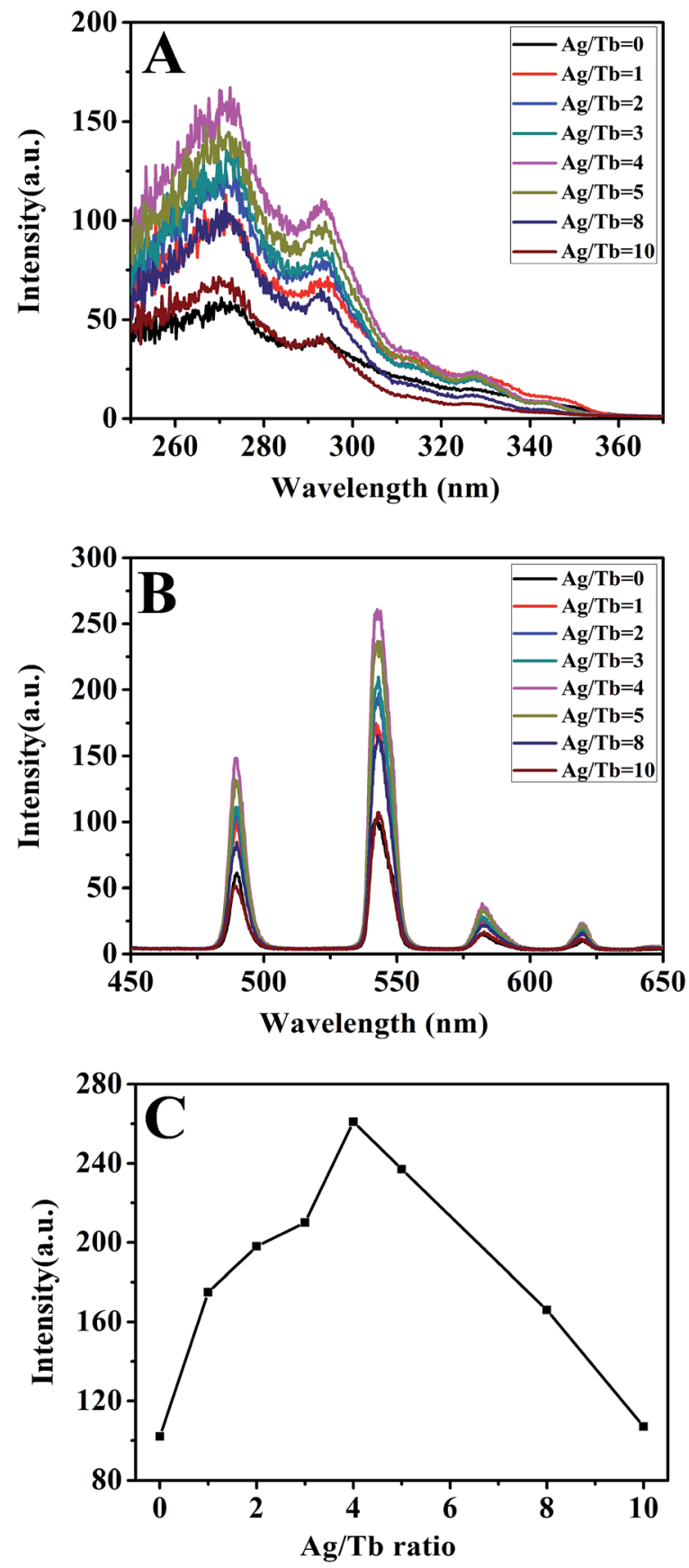

Fig. 6 (A) Excitation spectra $\left(\lambda_{\mathrm{em}}=539 \mathrm{~nm}\right)$, (B) emission spectra $\left(\lambda_{\text {ex }}\right.$ $=294 \mathrm{~nm}$ ) of Ag-NPs/Tb-complex/PLLA fibers with different Ag/Tb mole ratios and $(\mathrm{C})$ a plot of fluorescence intensity against $\mathrm{Ag} / \mathrm{Tb}$ mole ratios of the fibers.

confirms that Ag-NPs can enhance the fluorescence intensities of the Tb-complex/PLLA fibers. This positive effect is mainly attributed to LSPR of Ag-NPs. The LSPR effect on fluorescence property of fibers is illustrated in Fig. 7.

The intensity of LSPR can affect the fluorescence intensities of nearby rare earth ions. ${ }^{23} \mathrm{~A}$ high degree of the space locality of the LSPR of Ag-NPs leads to more significant enhancement on the surrounding local electromagnetic field close to Ag-NPs. ${ }^{31,32}$ The enhanced local electromagnetic field further leads to

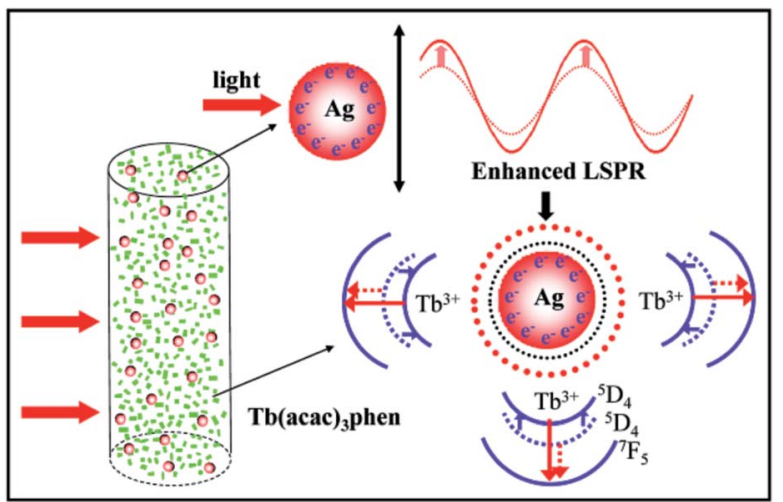

Fig. 7 Schematic diagram of LSPR effect on fluorescence of $\mathrm{Tb}^{3+}$ ions.

plasmonic interaction and also strongly affects the kinetic characteristics of nearby Tb-complex. ${ }^{33}$ Furthermore, the radiative decay rates of the $\mathrm{Tb}^{3+}$ are also enhanced. ${ }^{34}$ Meanwhile, the local field surrounding of $\mathrm{Tb}^{3+}$ ions is influenced. This has earlier been confirmed by the XPS analysis in Fig. 4. The change of local field surrounding further affects energy transfer between the ligands and $\mathrm{Tb}^{3+}$ ions and the resulting fluorescence intensity. As a result, the fluorescence intensity of fibers is greatly enhanced by Ag-NPs when the mole ratio of $\mathrm{Ag} / \mathrm{Tb}$ is $4: 1$. However, when the $\mathrm{Ag} / \mathrm{Tb}$ mole ratio further increases to $8: 1$, the intensity of the fibers decreases. The decrease in fluorescence intensity at a high concentration of Ag-NPs is attributed to the close distance between Ag-NPs and Tbcomplex, and the resulting fluorescence quenching of $\mathrm{Tb}^{3+}$ ions. ${ }^{22}$

Fluorescence quantum efficiency represents the ability of fluorescence materials transforming the absorbing light energy into fluorescence. Table 1 illustrates that Ag-NPs/Tb-complex/ PLLA fibers have higher quantum efficiency than the fibers without $\mathrm{Ag}$-NPs when the $\mathrm{Ag} / \mathrm{Tb}$ ratio is below 4 . This increase in quantum efficiency is also caused by the LSPR effect of Ag-NPs. When the $\mathrm{Ag} / \mathrm{Tb}$ ratio increases to 8 , the quantum efficiency of Ag-NPs/Tb-complex/PLLA fibers is lower than the fibers without Ag-NPs, and this is attributed to the fluorescence quenching by the excess Ag-NPs.

Fig. 8 shows the curve of the fluorescence decay for Ag-NPs/ Tb-complex/PLLA fibers. All of these curves could be well-fitted with the equation:

$$
I=A+B \exp (-t / \tau)
$$

where $I$ is the relative fluorescence intensity; $A$ and $B$ are the fitting constants, respectively; $t$ is the time; $\tau$ is fluorescence

Table 1 Quantum efficiencies $(\eta)$ and lifetimes $(\tau)$ of $\mathrm{Ag}-\mathrm{NPs} / \mathrm{Tb}-$ complex/PLLA fibers with different $\mathrm{Ag} / \mathrm{Tb}$ ratios

\begin{tabular}{lllll}
\hline Samples & $\begin{array}{l}\text { 1\# Ag/Tb } \\
=0\end{array}$ & $\begin{array}{l}2 \# \mathrm{Ag} / \mathrm{Tb} \\
=1\end{array}$ & $\begin{array}{l}3 \# \mathrm{Ag} / \mathrm{Tb} \\
=4\end{array}$ & $\begin{array}{l}4 \# \mathrm{Ag} / \mathrm{Tb} \\
=8\end{array}$ \\
\hline$\eta(\%)$ & 12.1 & 15.3 & 21.5 & 9.6 \\
$\tau(\mu \mathrm{s})$ & 1744 & 4063 & 9098 & 8252
\end{tabular}




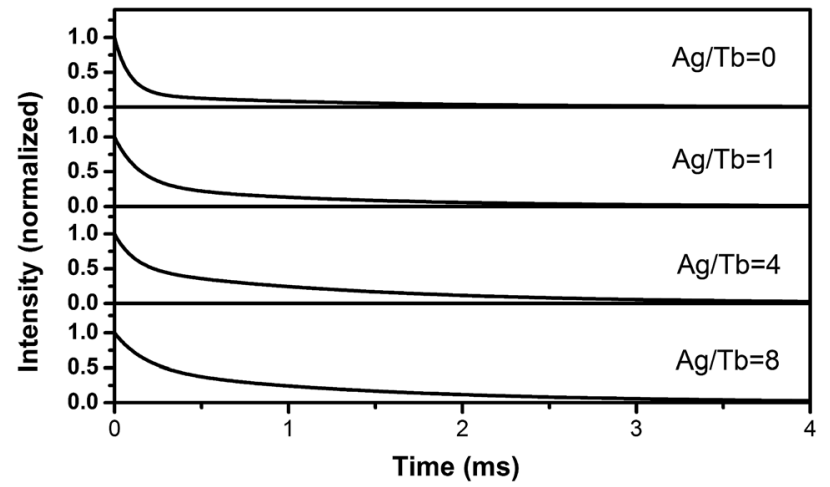

Fig. 8 Fluorescence decay curves of Ag-NPs/Tb-complex/PLLA fibers with different $\mathrm{Ag} / \mathrm{Tb}$ ratios.

lifetime. The measured values of the fluorescence lifetime are listed in Table 1. The fluorescence lifetimes of all Ag-NPs/Tbcomplex/PLLA fibers are higher than that of Tb-complex/PLLA fibers. According to the equation $\tau=1 /\left(A_{\text {rad }}+A_{\text {nrad }}\right){ }^{35}$ this enhancement of fluorescence lifetime is attributed to the decrease in the sum of the radiative transition rate $\left(A_{\mathrm{rad}}\right)$ and non-radiative transition rate $\left(A_{\text {nrad }}\right)$. As $A_{\text {rad }}$ is enhanced by the LSPR of Ag-NPs, the decrease in the $A_{\text {nrad }}$ mainly contributes to the decrease in the sum of $\left(A_{\mathrm{rad}}+A_{\text {nrad }}\right)$ and the resulting long lifetime of Ag-NPs/Tb-complex/PLLA fibers.

\section{Conclusions}

This work mainly prepared Ag-NPs/Tb-complex/PLLA fibers by electrospinning, and researched the enhanced fluorescence properties of the composite fibers. The results showed that the fibers had smooth surfaces and diameters of around $250 \mathrm{~nm}$. The Tb-complex dispersed uniformly in the PLLA fibers. With the addition of Ag-NPs, the local field surrounding environment of $\mathrm{Tb}^{3+}$ in the fibers was altered. Also, the LSPR effect of Ag-NPs influenced the energy transfer of Tb-complex, leading to an increase in fluorescence intensity of Ag-NPs/Tb-complex/PLLA fibers. Meanwhile, the addition of Ag-NPs improved quantum efficiency and extended fluorescence lifetime of $\mathrm{Tb}^{3+}$ in the composite fibers at the same time when the ratio of $\mathrm{Ag} / \mathrm{Tb}$ was below 4 .

\section{Acknowledgements}

The research was supported by the National Basic Research Program of China (2015CB654700 (2015CB674705)), the National Natural Science Foundation of China (51573007), the Fundamental Research Funds for the Central Universities in China (JD1513 and ZD1503).

\section{Notes and references}

1 M. C. Chen, Y. C. Sun and Y. H. Chen, Acta Biomater., 2013, 9, 5562-5572.

2 W. Liu, J. Wei, Y. Chen, P. Huo and Y. Wei, ACS Appl. Mater. Interfaces, 2013, 5, 680-685.
3 Z. Hou, G. Li, H. Lian and J. Lin, J. Mater. Chem., 2012, 22, 5254.

4 S. N. Raja, A. C. Olson, K. Thorkelsson, A. J. Luong, L. Hsueh, G. Chang, B. Gludovatz, L. Lin, T. Xu, R. O. Ritchie and A. P. Alivisatos, Nano Lett., 2013, 13, 3915-3922.

5 X. P. Zhang, S. P. Wen, S. Hu, Q. Chen, H. Fong, L. Q. Zhang and L. Liu, J. Phys. Chem. C, 2010, 114, 3898-3903.

6 Y. Lv, J. Wu, L. Wan and Z. Xu, J. Phys. Chem. C, 2008, 112, 10609-10615.

7 B. Sun, Y. Z. Long, Z. J. Chen, S. L. Liu, H. D. Zhang, J. C. Zhang and W. P. Han, J. Mater. Chem. C, 2014, 2, 1209-1219.

8 S. Wen, X. Zhang, L. Yao, M. Xi, L. Zhang, H. Fong and L. Liu, J. Phys. Chem. C, 2013, 1, 1613.

9 X. Zhang, S. Wen, S. Hu, L. Zhang and L. Liu, J. Rare Earths, 2010, 28, 333-339.

10 L. Huang, L. Cheng, H. Yu, J. Zhang, L. Zhou, J. Sun, H. Zhong, X. Li, Y. Tian, Y. Zheng, T. Yu, C. Li, H. Zhong, W. Liu, L. Zhang, J. Wang and B. Chen, Opt. Commun., 2012, 285, 1476-1480.

11 G. Gai, L. Wang, X. Dong and S. Xu, J. Mater. Sci., 2013, 48, 5140-5147.

12 C. F. Bohren and D. R. Huffman, Absorption and Scattering of Light by Small Particles, Wiley Interscience, 1983, vol. 98, pp. 290-291.

13 B. Sepúlveda, P. C. Angelomé, L. M. Lechuga and L. M. LizMarzán, Nano Today, 2009, 4, 244-251.

14 X. Liao, Y. Chen, M. Qin, Y. Chen, L. Yang, H. Zhang and Y. Tian, Talanta, 2013, 117, 203-208.

15 J. N. Anker, W. P. Hall, O. Lyandres, N. C. Shah, J. Zhao and R. P. V. Duyne, Nat. Mater., 2008, 7, 442-453.

16 R. Abargues, P. J. Rodriguez-Canto, S. Albert, I. Suarez and J. P. Martínez-Pastor, J. Mater. Chem. C, 2014, 2, 908-915.

17 O. L. Malta and M. A. Couto dos Santos, Chem. Phys. Lett., 1990, 174, 13-18.

18 R. Reisfeld, T. Saraidarov, G. Panzer, V. Levchenko and M. Gaft, Opt. Mater., 2011, 34, 351-354.

19 M. Eichelbaum and K. Rademann, Adv. Funct. Mater., 2009, 19, 2045-2052.

20 G. P. Acuna, F. M. Moller, P. Holzmeister, S. Beater, B. Lalkens and P. Tinnefeld, Science, 2012, 338, 506-510.

21 H. S. Wang, F. N. Xiao, Z. Q. Li, J. Ouyang, Z. Q. Wu, X. H. Xia and G. J. Zhou, Lab Chip, 2014, 14, 1123-1128.

22 H. S. Wang, C. Wang, Y. K. He, F. N. Xiao, W. J. Bao, X. H. Xia and G. J. Zhou, Anal. Chem., 2014, 86, 3013-3019.

23 H. Li, M. Wang, W. Qiang, H. Hu, W. Li and D. Xu, Analyst, 2014, 139, 1653-1660.

24 H. Lin, D. Chen, Y. Yu, R. Zhang and Y. Wang, Appl. Phys. Lett., 2013, 103, 091902.

25 T. Hayakawa, S. T. Selvan and M. Nogami, J. Non-Cryst. Solids, 1999, 259, 16-22.

26 D. Zhang, X. Hu, R. Ji, S. Zhan, J. Gao, Z. Yan, E. Liu, J. Fan and X. Hou, J. Non-Cryst. Solids, 2012, 358, 2788-2792.

27 X. Zong, K. Kim, D. Fang, S. Ran, H. S. Benjamin and C. Benjamin, Polymer, 2002, 43, 4403-4412.

28 W. K. Son, J. H. Youk, T. S. Lee and W. H. Park, Polymer, 2004, 45, 2959-2966. 
29 J. Bai, Y. Li, S. Yang, J. Du, S. Wang, J. Zheng, Y. Wang, Q. Yang, X. Chen and X. Jing, Solid State Commun., 2007, 141, 292-295.

30 Y. S. Kawashima, C. F. Gugliotti, M. Yee, S. H. Tatumi and J. C. R. Mittani, Radiat. Phys. Chem., 2014, 95, 91-93.

31 N. Calander and M. Willander, J. Appl. Phys., 2002, 92, 48784884.
32 P. Muhlschlegel, H. J. Eisler, O. J. Martin, B. Hecht and D. W. Pohl, Science, 2005, 308, 1607-1609.

33 C. Mihalcea, D. Büchel, N. Atoda and J. Tominaga, J. Am. Chem. Soc., 2001, 123, 7172-7173.

34 W. Ji, P. Jing and J. Zhao, J. Mater. Chem. C, 2013, 1, 470-476. 35 A. K. Bansal, A. Ruseckas, P. E. Shaw and I. D. W. Samuel, J. Phys. Chem. C, 2010, 114, 17864-17867. 